\title{
Influência do tratamento por realidade virtual no equilíbrio de um paciente com paralisia cerebral
}

\section{Influence of virtual reality treatment for balance in a patient with cerebral palsy}

\author{
Gleyson Luiz Bezerra Lopes ${ }^{1}$, Kim Mansur Yano ${ }^{1}$, Nathália Stéphany Araújo \\ Tavares $^{2}$, Isabelle Ananda de Oliveira Rego ${ }^{2}$, Robson Inácio $\mathrm{Marinho}^{2}$, Luciana \\ Protásio de $\mathrm{Melo}^{3}$, Karyna Myrelly Oliveira Bezerra de Figueiredo Ribeiro ${ }^{4}$, Fabrícia \\ Azevedo da Costa Cavalcanti ${ }^{5}$
}

http://dx.doi.org/10.11606/issn.2238-6149.v24i2p121-126

Lopes GLB, Yano KM, Tavares NSA, Rego IAO, Marinho RI, Melo LP, Ribeiro KMOBF, Cavalcanti FAC. Influência do tratamento por realidade virtual no equilíbrio de um paciente com paralisia cerebral. Rev. Ter. Ocup. Univ. São Paulo, 2013 maio/ago, 24(2);121-6.

RESUMO: A melhora da capacidade de manutenção do equilíbrio é essencial para quem apresenta algum comprometimento motor, como a Paralisia Cerebral (PC). A realidade virtual (RV) já se destaca na reabilitação neurológica, porém com poucos estudos que abordem pacientes adultos com PC. Este estudo teve como objetivo investigar a influência da RV por meio de jogos de vídeo Wii no equilíbrio em posição ortostática de um paciente com PC Espástica Hemiparética. Foi utilizado o equipamento Nintendo ${ }^{\circledR}$ Wii, com o jogo Wii Fit Plus, em treinamentos que estimularam o equilíbrio látero-lateral e ântero-posterior do paciente. Os instrumentos aplicados foram: Escala de Equilíbrio de Berg, Medida de Independência Funcional, além de 3 testes do Neurocom Balance Master: Limits of Stability, Tandem Walk e Step/Quick Turn. O protocolo de tratamento consistiu de 10 sessões, 30 minutos cada, duas vezes por semana por 38 dias. Ao final foi possível observar um aumento expressivo na pontuação de vários itens dos testes realizados, implicando na melhora da capacidade de equilíbrio do paciente. Esses resultados sugerem a influência benéfica do Wii no que concerne o equilíbrio e capacidade funcional do portador de $\mathrm{PC}$, tornando-o mais confiante para realizar suas atividades diárias.

PALAVRAS-CHAVES: Modalidades de Fisioterapia; Reabilitação; Equilíbrio Postural; Terapia de Exposição à Realidade Virtual.
Lopes GLB, Yano KM, Tavares NSA, Rego IAO, Marinho RI, Melo LP, Ribeiro KMOBF, Cavalcanti FAC. Influence of virtual reality treatment for balance in a patient with cerebral palsy. Rev. Ter. Ocup. Univ. São Paulo, 2013 maio/ago, 24(2);121-6.

\begin{abstract}
The improvement of maintaining postural balances its essential $\mathrm{i}$ for those who have some motor impairment, such as Cerebral Palsy (CP). Virtual Reality (VR) is a feature that is standing out in neurological rehabilitation, but there are few studies regarding adult patients with $\mathrm{CP}$. This study aimed to investigate the influence of the VR through the Wii on balance in standing position from a patient with Spastic Hemiplegic CP. It was used the equipment Nintendo ${ }^{\circledR}$ Wii, with the game Wii Fit Plus, where the training chosen stimulated the latero-lateral and anterior-posterior patient's balance. The instruments used were: Berg Balance Scale, Functional Independence Measure, and 3 Neurocom Balance Master Systems tests: Limits of Stability, Tandem Walk and Step/ Quick Turn. The treatment protocol consisted of 10 sessions, with 30 minutes each, performed twice a week for 38 days. At the end of treatment, a significant increase in the scores of various test items performed was observed, which resulted in an improvement of patient's balance. Results suggest a beneficial influence of the video game Wii regarding to postural balance and functional capacity of patients with PC, which become more confident to carry out their activities of daily living.
\end{abstract}

KEYWORDS: Physical Therapy Modalities; Rehabilitation; Postural Balance; Virtual Reality Exposure Therapy.

\footnotetext{
Este artigo foi o trabalho de conclusão de curso do fisioterapeuta Gleyson Luiz Bezerra Lopes e parte integrante do projeto Realidade Virtual no Contexto da Reabilitação de Pacientes, do departamento de Fisioterapia/UFRN.

O resumo deste artigo foi apresentado no IV Congresso Anual de Saúde da Universidade de Aveiro com o tema "do gene a degeneração", nos dias 13 e 14 de abril de 2013, em Aveiro, Portugal.

${ }^{1}$.Fisioterapeuta formado pela Universidade Federal do Rio Grande do Norte/UFRN.

2.Aluno(a) do curso de Fisioterapia da Universidade Federal do Rio Grande do Norte/UFRN.

${ }^{3}$ Fisioterapeuta. Doutoranda em Fisioterapia/UFRN e docente do curso de Fisioterapia da Faculdade Estácio do Rio Grande do Norte. protasio.melo@yahoo.com.br

4.Fisioterapeuta. Doutoranda em Ciências da Saúde/UFRN. karynafigueiredo@ufrnet.br

5. Fisioterapeuta. Doutora em Ciências da Saúde/UFRN e docente do curso de Fisioterapia da UFRN. fabriciacosta@ufrnet.br Endereço para correspondência: Luciana Protásio de Melo. Rua Estrela do Mar, 222. Bloco Brasil, apto 304. Nova Parnamirim. Parnamirim, RN. CEP: 59.151-120.
} 


\section{INTRODUÇÃO}

$\mathrm{P}$ aralisia Cerebral (PC) pode ser definida como uma desordem de movimento e postura devido a um defeito ou lesão do encéfalo em desenvolvimento ${ }^{1}$. Dentre suas possíveis causas se pode citar as infecções maternas durante o primeiro e o segundo trimestre da gravidez, como rubéola, citomegalovírus e toxoplasmose ${ }^{2}$. Alguns fatores como a hipóxia pré-parto, doença de Von Willebrand, medicações específicas, abuso de álcool e drogas ilícitas e traumatismos abdominais graves ${ }^{3}$, além da má formação congênita incluindo má-formação no desenvolvimento cortical ${ }^{2}$, também estão associados à prevalência da PC.

O problema motor dos indivíduos com PC originase de disfunção do sistema nervoso central, que interfere diretamente no desenvolvimento do controle postural contra gravidade e impede o desenvolvimento motor normal ${ }^{4}$. A disfunção no controle postural interfere nas atividades funcionais diárias, sendo um dos problemas principais nessa populaçãa $0^{5}$.

Os déficits na PC incluem anormalidades do tônus muscular, do equilíbrio e da força muscular, além de ser comum o aparecimento de contraturas musculares e deformidades ${ }^{6}$, o que leva a um atraso no desenvolvimento do controle motor e nas respostas posturais ${ }^{7}$. Dessa forma, o controle postural, que é a função fundamental do controle motor e que está envolvido em muitas funções motoras, especialmente na marcha, também se encontra debilitado8.

$\mathrm{O}$ curso natural da PC mudou bastante durante os últimos 50 anos. Estudos em vários países têm demonstrado que a expectativa de vida de indivíduos com PC tem aumentado?.

Pesquisas sobre os aspectos relacionados à saúde de adultos com PC são importantes para que profissionais da saúde possam disponibilizar abordagens terapêuticas adequadas e documentar a necessidade de políticas públicas voltadas para essa população ${ }^{10}$. Com tal propósito, nos últimos anos, há um fluxo crescente de estudos sobre adultos com $\mathrm{PC}^{9}$.

A fisioterapia assume papel crucial na abordagem multidisciplinar no processo de reabilitação. Assim, encontrar novas formas e programas de reabilitação é essencial para aumentar a motivação e envolvimento do paciente. Nesta cena, a Realidade Virtual (RV) vem sendo amplamente utilizada como uma ferramenta de auxílio na área da neuroreabilitação ${ }^{11}$.

A RV consiste numa tecnologia computadorizada que simula elementos e ações da vida real, possibilitando uma interação complexa entre paciente e máquina promovendo, assim um feedback sensorial, cognitivo, psicológico e motor $^{12}$. Devido a esse ambiente lúdico e atraente, de onde muitas vezes o paciente não deseja sair, ele executa ações de maneira sistemática e repetitiva, porém sem perceber, sendo o propósito da terapia mais rapidamente alcançado ${ }^{11}$.

Nesse sentido, um instrumento de RV que tem sido bastante difundido na área da medicina de reabilitação é o vídeo game Nintendo ${ }^{\circledR}$ Wii, que vem se destacando como um simulador da realidade, especialmente pelo jogo Wii Fit Plus $^{\circledR}$ e do acessório Balance Board ${ }^{\circledR}$, que tem comprovada sua viabilidade, segurança e eficácia na melhora do equilíbrio em diversos estudos já realizados ${ }^{13}$.

Ademais há o limitado número de pesquisas sobre o tema, o que é justificável por seu pouco tempo de existência. Há necessidade de pesquisas com rigor metodológico visando à segurança, eficácia e validade da RV no ambiente terapêutico.

Neste estudo, um paciente adulto com PC, que se enquadrava como funcionário da instituição e era acompanhado no Programa de Prevenção ao Estresse do departamento de Fisioterapia, foi submetido a um protocolo de reabilitação entre outubro e novembro/2012 com o jogo Wii Fit plus ${ }^{\circledR}$, área pouco explorada pela RV, com o objetivo de analisar seus efeitos na melhora do equilíbrio.

\section{MÉTODOS}

A pesquisa realizada é um estudo de caso no qual contou com a participação de um paciente com diagnóstico clínico de Paralisia Cerebral Espástica Hemiparética.

Para inclusão na pesquisa, admitiram-se os seguintes critérios: diagnóstico de Paralisia Cerebral; ser adulto e apresentar déficit de equilíbrio (de acordo com o teste Limits of Stability do aparelho Balance Master ${ }^{\circledR}$ da NeuroCom International Inc ${ }^{\circledR}$ ). Admitiu-se como critério de exclusão a desistência do projeto ou a não adaptação ao jogo.

$\mathrm{Na}$ coleta dos dados gerais do paciente, utilizou-se Ficha de Avaliação Fisioterapêutica para preenchimento de informações como: nome completo, sexo, idade, estado civil, diagnóstico, tempo decorrido de patologia, escolaridade, patologias associadas, demais comorbidades e histórico familiar, dentre outros.

$\mathrm{Na}$ avaliação funcional objetiva do equilíbrio, utilizou-se o equipamento Balance Master ${ }^{\mathbb{B}}$ da NeuroCom International Inc ${ }^{\circledR}$, do Departamento de Fisioterapia da UFRN. O instrumento possui duas plataformas de força adjacentes conectadas a um computador no qual é capaz de detectar as oscilações no centro de gravidade (COG) dentro de um limite de estabilidade predefinido (LOS) ${ }^{14}$. $\mathrm{O}$ sistema provê medidas quantitativas da performance do equilíbrio estático e dinâmico e feedback visual da excursão e posição do $\mathrm{COG}^{14}$. Para avaliação do equilíbrio dinâmico, foi realizado o teste do Limits of Stability, que quantifica a 
distância máxima que uma pessoa pode intencionalmente deslocar seu centro de gravidade (CG), ou seja, inclinar seu corpo em uma determinada direção, sem perder o equilíbrio, dar passos, ou precisar de ajuda. Foi aplicado também o teste do Tandem Walk, que quantifica características da marcha enquanto o paciente anda alinhado e aproximando o calcanhar ao dedo do pé, a partir de uma extremidade da placa de força até a outra, permanecendo parado ao finalizar o percurso. E foi feito também o teste Step/Quick Turn, que avalia o equilíbrio dinâmico medido pela oscilação do COG durante o giro, além do tempo gasto para realizá-lo. Para realizar o teste, o paciente caminhou sobre a plataforma, iniciando a marcha com o membro esquerdo, e em seguida realizou um giro de 180 graus para o lado esquerdo, sem perder o contato com o solo. Após as três tentativas para o lado esquerdo, o paciente realizou com o lado direito, isto é, iniciar a marcha com o membro direito e realizar o giro para o mesmo lado $^{14}$.

Também se aplicou a versão brasileira da Medida de Independência Funcional (MIF) por ser uma medida que atende a critérios de confiabilidade, validade, precisão, praticidade e facilidade ${ }^{15}$. Neste estudo, foi contemplada somente a MIF motora, com pontuação mínima de $13 \mathrm{e}$ máxima de 91 pontos $^{16}$.

Em associação aos instrumentos mencionados, aplicou-se a versão brasileira da Escala de Equilíbrio de Berg (EEB), para avaliação do equilíbrio em condições estáticas e dinâmicas por meio de 14 situações. A pontuação máxima a ser alcançada é de 56 pontos.

\section{Relato de caso}

Paciente do sexo masculino, 25 anos, solteiro, com ensino superior completo, foi diagnosticado com PC por volta dos 6 meses de idade.

Ao exame físico foram observados vários comprometimentos posturais, como hiperlordose lombar, escoliose a nível toraco-lombar com curvatura para a direita e gibosidade à esquerda, posteriorização de cabeça com inclinação para a esquerda e leve rotação para a direita. Possui hemiparesia espástica de leve a moderada à esquerda. Realizou tratamento fisioterapêutico contínuo até os 5 anos, alternando períodos com e sem acompanhamento da fisioterapia a partir dessa idade. No período da realização do estudo, fazia fisioterapia domiciliar duas vezes por semana, para alívio das dores na coluna. Para a realização da pesquisa, foi solicitado ao fisioterapeuta domiciliar que limitasse o tratamento somente para o alívio da dor, com o intuito de não interferir nos resultados finais.

\section{Procedimento de coleta de dados}

A pesquisa foi submetida ao Comitê de Ética em
Pesquisa da UFRN e aprovado em 13 de setembro de 2012, de acordo com a Resolução 196/96 sob número do parecer 97.254. Após a assinatura do Termo de Consentimento Livre e Esclarecido (TCLE), o paciente passou por uma avaliação fisioterapêutica pré-protocolo e outra pós-protocolo de tratamento.

O protocolo de tratamento consistiu de 2 atendimentos fisioterapêuticos por semana, com duração de 30 minutos cada, totalizando 11 sessões, sendo considerada uma falta, assumindo-se então 10 sessões.

Os atendimentos foram realizados por dois fisioterapeutas e decorreram da seguinte forma: inicialmente foi aferida a pressão arterial (PA) do paciente, em seguida aplicou-se o protocolo de tratamento com a RV, que consistiu de 20 minutos de terapia, e ao final a PA era novamente aferida. Os 10 minutos restantes ficaram reservados para as aferições de pressão e para intervalos de descanso do paciente, caso necessário.

Foi utilizado um vídeo game da marca Nintendo ${ }^{\circledR}$, denominado Wii, e o jogo atribuído a esse protocolo foi o Wii Fit plus. Esse jogo possui um acessório chamado Balance Board, sendo ele responsável pela interface entre máquina e jogador ${ }^{17}$.

Dentre os possíveis jogos para essa modalidade, foram selecionados sete, sendo que o protocolo inicial era formado por 5 jogos, tendo 2 jogos de reserva, destinados a substituir os jogos iniciais, como forma de evolução do tratamento. Os jogos elegidos foram: Balance Bubble: para fazê-lo, o paciente/avatar necessita se deslocar em todas as direções; Tightrope: durante o jogo a marcha deve ser simulada alternando os pés sobre a balança. Há também simulação de saltos com um rápido movimento de flexoextensão dos joelhos; Soccer Heading: o paciente deve fazer movimentos latero-laterais na balança a fim de cabecear o maior número de bolas e se esquivar de objetos jogados em sua direção. Penguin Slide: neste jogo o paciente movimentase látero-lateralmente visando que o avatar pegue o maior numero de peixes; Table Tilt: jogo com finalidade de acertar as bolas dispostas numa plataforma em buracos. Para mover a plataforma, comandada pelo paciente, esse tem que se deslocar em todas as direções (frente, trás, laterais); Ski Jump: jogador mantém o avatar estável sobre esquis no gelo e precisa simular um salto, partindo de uma flexão de joelhos para uma extensão (esse jogo substitui o Tightrope na $7^{\mathrm{a}}$ sessão); Ski Slalom: o paciente faz movimentos para esquerda e direita (latero-lateralmente) a fim de passar entre várias bandeiras (esse jogo substitui o Soccer Heading na $8^{\text {a }}$ sessão).

Os jogos descritos visam o treino dos ajustes posturais ântero-posteriores e látero-laterais e do controle do equilíbrio através da interação entre paciente e $\mathrm{jogo}^{17}$. Cada jogo foi realizado 3 vezes ou por no máximo 5 minutos 
ininterruptos.

O paciente não recebeu nenhum outro treinamento de equilíbrio durante o processo de tratamento. Para sua segurança, além de dois fisioterapeutas ao seu lado, foi utilizado um andador localizado na altura de sua cintura, para o caso de desequilíbrio na realização do protocolo de RV.

\section{RESULTADOS}

Ao final das 10 sessões, verificaram-se alterações positivas no equilíbrio e controle de tronco do participante. O paciente referiu melhora nas atividades de vida diárias (AVDs) principalmente para subir e descer escadas.

Dentre as sessões não houve intercorrência, somente um intervalo para descanso de 2 a 4 minutos entre o quarto e o quinto jogo. A PA do paciente manteve-se entre 90 e 120 $\mathrm{mmHg}$ (sistólica) e 60 e $90 \mathrm{mmHg}$ (diastólica) durante todo o programa.

Após a $4^{\mathrm{a}}$ sessão, nos jogos Balance Bubble e Tightrope, o paciente já era capaz de controlar seu avatar de forma visivelmente mais estável e segura durante todo o percurso. Na $7^{\text {a }}$ sessão o jogo Ski Jump substituiu o Tightrope, e então o desempenho do paciente no Penguin Slide melhorou notavelmente. $\mathrm{Na} 8^{\mathrm{a}}$ sessão o Soccer Heading foi substituído pelo Ski Slalom, notando-se uma melhor adaptação a este jogo.

No início do tratamento, o paciente recebia auxílio dos terapeutas para correções posturais a fim de otimizar a sua adaptação aos jogos. Esse estímulo foi sendo diminuído progressivamente, de forma que o paciente terminou os atendimentos sem nenhum auxílio durante os jogos.

Após a finalização do protocolo de atendimento, foi possível observar que não houve alteração no escore da MIF motora, que permaneceu em 86, onde apenas os itens "alimentação" (escore 5) e "vestir a metade inferior do corpo" (escore 4) apresentaram-se com nível de dependência.

Já na EEB, observou-se um acréscimo de 6 pontos entre as avaliações inicial e final, de 48 para 54. No item "permanecer em pé com os olhos fechados", o escore passou de 1 para 4; tanto em "posicionar os pés alternadamente no degrau" como em "permanecer em pé com um pé à frente", o paciente evoluiu de 3 para 4; e no item "permanecer em pé sobre um pé", sua pontuação passou de 1 para 2.

No teste Limits of Stability (LOS), a média do "tempo de reação" caiu de 0,96 s para 0,39 s, a média de "velocidade do movimento" aumentou de 4,2 graus/s para 6,1 graus/s, a média das distâncias do ponto final/alcance máximo progrediu de $57 / 75 \%$ para $72 / 85 \%$, e o "controle direcional" do movimento evoluiu de $47 \%$ para $61 \%$.

No Tandem Walk, a largura do passo diminuiu de $16,2 \mathrm{~cm}$ para $9,7 \mathrm{~cm}$, tendo ainda aumentado a velocidade de $15 \mathrm{~cm} / \mathrm{s}$ para $27,7 \mathrm{~cm} / \mathrm{s}$, e diminuído sua oscilação no ponto final de 8,6 graus/s para 3,5 graus/s. Finalizando, os comparativos do Step/Quick Turn antes e após o protocolo também apresentaram diferença, tendo diminuído o tempo de giro para direita/esquerda, de 4,37/4,36s para 1,69/3,85s, além da oscilação durante o giro para direita/esquerda, de $89,3 / 57,8$ graus para $31,2 / 38,8$ graus.

Segundo os manuais do Neurocom Balance Master, todas essas alterações de dados, sem exceção, indicam mudanças positivas para o paciente, evidenciando adaptações posturais mais rápidas e precisas, traduzidos em um controle motor mais eficaz ${ }^{16}$. A Tabela 1 apresenta todos os dados citados.

Tabela 1 - Pontuação das variáveis avaliadas nos testes do paciente (pré e pós-protocolo)

\begin{tabular}{|c|c|c|c|}
\hline & Antes & Depois & Diferença \\
\hline MIF & 119 & 119 & 0 \\
\hline Cognitiva & 33 & 33 & 0 \\
\hline Motora & 86 & 86 & 0 \\
\hline BERG & 48 & 54 & 6 \\
\hline Permanecer em pé com os olhos fechados & 1 & 4 & 3 \\
\hline Posicionar os pés alternadamente no degrau & 3 & 4 & 1 \\
\hline Permanecer em pé com um pé á frente & 3 & 4 & 1 \\
\hline Permanecer em pé sobre um pé & 1 & 2 & 1 \\
\hline \multicolumn{4}{|l|}{$\begin{array}{l}\text { LIMITS OF STABILITY } \\
\end{array}$} \\
\hline Tempo de reação (s) & 0,96 & 0,39 & $-0,57(59 \%)$ \\
\hline Velocidade de movimento (graus/s) & 4,2 & 6,1 & $+1,9(45 \%)$ \\
\hline Ponto final / Alcance máximo (\%) & $57 / 75$ & $72 / 85$ & $+15 / 10(26 / 13 \%)$ \\
\hline Controle direcional $(\%)$ & 47 & 61 & $+14(29 \%)$ \\
\hline \multicolumn{4}{|l|}{ TANDEM WALK } \\
\hline Largura do passo $(\mathrm{cm})$ & 16,2 & 9,7 & $-6,5(40 \%)$ \\
\hline Velocidade $(\mathrm{cm} / \mathrm{s})$ & 15 & 27,7 & $+12,7(84 \%)$ \\
\hline Oscilação no ponto final (graus/s) & 8,6 & 3,5 & $-5,1(59 \%)$ \\
\hline \multicolumn{4}{|l|}{ STEP/QUICK TURN } \\
\hline Tempo de giro direita/esquerda (s) & $4,37 / 4,36$ & $1,69 / 3,85$ & $-2,68 / 0,51(61 / 11 \%)$ \\
\hline Oscilação durante o giro direita/esquerda (graus) & $89,3 / 57,8$ & $31,2 / 38,8$ & $-58,1 / 19(65 / 32 \%)$ \\
\hline
\end{tabular}




\section{DISCUSSÃO}

Observando a maior sobrevida dos pacientes com algum comprometimento neurológico, torna-se extremamente necessário o desenvolvimento de instrumentos que promovam saúde e qualidade de vida para essa população ${ }^{13}$.

O sistema Wii Fit permite o tratamento de disfunções motoras relacionadas ao equilíbrio, permitindo adaptações às limitações do paciente ${ }^{18}$. Esse sistema apresenta aos pacientes muitos feedbacks extrínsecos, como o auditivo através de alto-falantes; o visual por meio de sobreposições na tela, o feedback háptico representado por vibração e controle de elementos na tela pelo Wii Remote ${ }^{19}$.

Considerando isso, os jogos permitem o treinamento de controle do equilíbrio por meio de diversas situações de instabilidade, solicitando do paciente transferências de peso médio-laterais e anteroposteriores, fundamentais para a estabilidade dinâmica de tronco, e assim levando a melhora na capacidade de controle motor, equilíbrio estático e dinâmico, $\mathrm{e}, \log$, na capacidade funcional.

Estudo $^{20}$ realizado com o Wii Balance Board verificou a funcionalidade da correção da postura em pé. Foi avaliado se duas pessoas com múltiplas deficiências seriam capazes de corrigir a sua postura ativa em pé de acordo com a estimulação. Os dados mostraram que ambos os participantes aumentaram significativamente a duração do tempo de manter a postura correta em pé, ativando assim o sistema de controle.

Os efeitos da RV como instrumento de reabilitação principalmente sobre o equilíbrio em pacientes neurológicos já foram documentados em diversos trabalhos. Um estudo do tipo experimental, cego e randomizado envolvendo 10 pacientes com $A V C$ crônico sugeriu que a $R V$ poderia induzir uma reorganização cortical para recuperação motora desses pacientes ${ }^{21}$.

Estudo recente ${ }^{13}$ avaliou os efeitos de uma intervenção com o Nintendo Wii no equilíbrio de pacientes hemiparéticos e demonstrou o aumento na capacidade de controle do equilíbrio estático e dinâmico, pela análise de escores da escala de Berg.

Vários estudos que utilizaram protocolos com o Wii Fit vêm demonstrando melhora não só do equilíbrio, mas também no aumento da capacidade funcional, traduzido em ajustes posturais mais eficientes durante a execução de atividades ${ }^{13,22}$. Essa evolução pode estar relacionada com a melhora significativa nos índices de tempo de reação, velocidade e controle direcional dos movimentos, encontradas neste estudo, uma vez que estão diretamente relacionados com a precisão do controle motor $^{23}$.

Além disso, outro reflexo do aprimoramento do controle motor é uma menor oscilação do COG durante os movimentos. Um estudo de caso ${ }^{22}$ com um paciente de PC demonstrou que, após a aplicação do protocolo com o Wii Fit, foi possível perceber uma diminuição de $60 \%$ na variação do COG durante a marcha. Fato que também está de acordo com os resultados aqui encontrados no LOS e no Step/Quick Turn.

Os dados positivos com relação à melhora no controle motor em pacientes neurológicos são corroborados pelo presente estudo uma vez que houve melhora quantitativa em vários parâmetros relacionados ao equilíbrio postural mensurados com o Balance Master System, como um aumento na velocidade do movimento, maior controle direcional e alcance final nos limites de estabilidade; diminuição da largura do passo e menor oscilação ao término da caminhada em tandem e execução durante o teste tandem walk com maior velocidade; realização do giro com um menor tempo, tanto para direita quanto esquerda associado a uma menor oscilação durante o giro no teste do step/ quick turn.

Muito embora a MIF motora não tenha sofrido alteração neste estudo, partindo da análise dos resultados dos demais instrumentos de avaliação, pelo relato do paciente houve ganhos funcionais, traduzidos em segurança e qualidade dos movimentos realizados previamente por ele.

O processo de reabilitação de pacientes neurológicos é, muitas vezes, um desafio para o terapeuta, pois é um processo que requer o máximo esforço do paciente. Para que este processo possa ser executado da melhor forma, é necessário que o indivíduo esteja motivado, para tal, o terapeuta deve diversificar os exercícios de modo que consiga estimular e motivar o indivíduo para sua recuperação ${ }^{19,24}$. O sistema de jogos Wii foge dos exercícios fisioterapêuticos convencionais, muitas vezes monótonos e repetitivos, proporcionando desafios novos ao paciente com maior motivação.

\section{CONCLUSÃO}

Os resultados obtidos no presente trabalho demonstram que o treino de equilíbrio usando o Wii Fit Plus parece proporcionar resultados importantes na reabilitação fisioterapêutica do portador de PC. Tais resultados confirmados pelo sistema preciso do Balance Master System ${ }^{\circledR}$ da NeuroCom International Inc ${ }^{\circledR}$.

Além disso, este estudo demonstrou a fácil aplicabilidade deste recurso de tratamento, o qual, além de estimular os sistemas sensorial, motor e cognitivo do paciente, ofereceu um alto grau de motivação e adesão à terapia.

Por fim, baseando-se na resposta e desempenho do paciente, sugere-se que os jogos Balance Bubble, Penguin Slide, Table Tilt, Sky Jump e Ski Slalom são os mais 
Lopes GLB et al. Influência do tratamento por realidade virtual. Rev. Ter. Ocup. Univ. São Paulo, 2013 maio/ago, 24(2);121-6.

indicados para o treinamento de equilíbrio em pacientes com PC, porém ainda necessitamos de mais estudos para sua

\section{REFERÊNCIAS}

1. Rosembeaum P, Paneth N, Leviton A, Goldstein M, Bax M, Damiano D, et al. A report: the definition and classification of cerebral palsy. Dev Med Child Neurol. 2007;49(6):8-14.

2. Reddihough, D.S.; Collins, K.J. The epidemiology and causes of cerebral palsy. Aust J Physiother. 2003;49(1):7-12.

3. Wong JP, Seow WT, Yeo GS. Characteristics of Six Newborn Infants with Postnatal Findings of Severe Intracranial Haemorrhage. Ann Acad Med, Singapore. 2004;33(6):789-92.

4. Howle JM. Neuro-developmental treatment approach: theoretical foundations and principles of clinical practice. Laguna Beach, CA: Neuro Development Treatment Association; 2003.

5. Brogren E, Algra MH, Forssberg H. Postural control in sitting children with cerebral palsy. Neurosci Biobehav Rev. 1998;22(4):591-96.

6. Papavasiliou AS. Management of motor problems in cerebral palsy: A critical update for the clinician. Eur J Paediatr Neurol. 2009;13(5):387-96.

7. Palmer FB. Strategies for the early diagnosis of cerebral palsy. J Pediatr. 2004;145(2):8-11 [cited Sep 10]. Available from: http:/ www.pedclerk.sites.uchicago.edu/sites/pedclerk.uchicago.edu/ files/uploads/strat.pdf.

8. Hadders-Agra M, Brogren E, Forssberg H. Ontogeny of postural adjustments during sitting in infancy: variation, selection and modulation. J Physiol. 1996;493:273-88 [ciited 2012 Sep 15]. Available from: http://www.ncbi.nlm.nih.gov/pmc/articles/ PMC1158968/.

9. Strauss D, Brooks J, Rosenbloon L, Shavelle R. Life expectancy in cerebral palsy: an update. Dev Med Child Neurol. 2008;50(7):487-93.

10. Bottos M, Gericke C. Ambulatory capacity in cerebral palsy: prognostic criteria and consequences for intervention. Dev Med Child Neurol. 2003;45(11):786-90.

11. Vaghetti CA, Botelho SS. Ambientes virtuais de aprendizagem na educação física: uma revisão sobre a utilização de exergames. Ciên Cogn. 2010;15(1):76-88.

12. Saposnik G, Mandani M, Bayley M, Thorpe KE, Hall J, Cohen LG, Teasell R. Effectiveness of Virtual Reality Exercises in Stroke Rehabilitation (EVREST): Rationale, Design, and Protocol os a Pilot Randomized Clinical Trial Assessing the Wii Gaming System. Int J Stroke. 2010;5(1):47-51 [cited comprovação, sendo o jogo a ser escolhido pelo terapeuta um dos maiores desafios na reabilitação com RV.

2012 Oct 20]. Available from: <http://works.bepress.com/cgi/ viewcontent.cgi? article $=1001 \&$ context $=$ gustavo_saposnik.

13. Barcala L, Colella F, Araujo MC, Salgado ASI, Oliveira CS. Análise do equilíbrio em pacientes hemiparéticos após o treino com o programa Wii Fit. Fisioter Mov. 2011;24(2):337-43.

14. Neurocom ${ }^{\circledR}$ International Inc. Balance Master Operators Manual. 8.3 version. Clackamas; 2000.

15. Hamilton BB, Laughlin JA, Granger CV, Kayton RM. Interrater agreement of the seven-level Functional Independence Measure (FIM). Arch Phys Med Rehabil. 1991;72:790.

16. Riberto M, Miyazaki MH, Juca SSH, Sakamoto H, Pinto PPN, Battistella LR. Validação da Versão Brasileira da Medida de Independência Funcional. Acta Fisiátr. 2004;11(2):72-6 [citado 10 out. 2012]. Disponível em: http://www.unifra.br/professores/ anabonini/Valida $\% \mathrm{C3} \% \mathrm{~A} 7 \% \mathrm{C} 3 \% \mathrm{~A} 3 \mathrm{o} \% 20$ Brasileira $\% 20 \mathrm{MIF}$. pdf.

17. Schiavinato AM, Baldan C, Mellato L, Lima LS. Influência do Wii Fit no equilíbrio de paciente com disfunção cerebelar: estudo de caso. J Health Sci Inst. 2010;28(1):50-2.

18. Sveitrupp H. Motor rehabilitation using virtual reality. J Neuro Eng Rehabil. 2004;1:1-8.

19. Anderson F, Annett M, Bischof W. Lean on Wii: physical rehabilitation with virtual reality and Wii peripherals. Annu Rev Cyber Ther Telemed, San Diego, CA. 2010;8:181-4.

20. Shih CH, Shih CT, Chu CL. Assisting people with multiple disabilities actively correct abnormal standing posture with a Nintendo Wii Balance Board through controlling environmental stimulation. Res Dev Disabil. 2010;31(4):936-42.

21. Ching HS, Man LC, Ching TS. A limb action detector enabling people with multiple disabilities to control environmental stimulation through limb action with a Nintendo Wii remote controller. Res Dev Disabil. 2010;31(5):1047-53.

22. Teixeira CS, Alves RF, Pedroso FS. Sistema estomatognático postura e equilíbrio corporal. Salusvita, Bauru. 2010;29(2):69-81.

23. You SH, Jang SH, Kim YH, Hallet M, Ahn SH, Kwon YH, Kim JH, Lee MY. Virtual Reality-induced cortical reorganization and associated locomotor recovery in chronic stroke: an experimenterblind randomized study. Stroke. 2005;36(7):1166-71.

24. Braga M. Realidade virtual e educação. Rev Biol Ciênc Terra. 2001;1(1):1-8. 